Nature Reviews Genetics | AOP, published online 14 July 2009; doi:10.1038/nrg2646

$\Rightarrow$ TECHNOLOGY

\title{
The sequencing game
}

A new approach to pooling DNA samples promises to increase the number of templates that can be sequenced in a single experiment from a few dozen to over 100,000. This 'DNA Sudoku' method, named after the logic puzzle, will offer substantial cost savings to high-throughout sequencing and efficient medical genotyping.

The pooling of DNA sequencing samples is not new, but current protocols rely on bar coding each sample with a short oligonucleotide, which is then used to associate a read to the correct sample. This approach is laborious, however, as a unique tag has to be created for each sample. The new method creates pools of samples, and then associates a bar code to each pool, rather than to each individual sequence. Because the samples are pooled in a particular pattern, a Sudoku-like approach is needed to assign each read to a particular pool, and then to a particular sample. The reliability of the method $(97 \%)$ was established in silico and in two experimental trials: to detect cystic fibrosis carrier mutations in 18,000 individuals and the presence of particular short hairpin RNA inserts in over 40,000 bacterial clones.

Because new-generation sequencing technologies produce only short reads, DNA Sudoku is most useful in detecting the presence of medically relevant mutations in defined genomic regions; although Prabhu and Pe'er also describe a further use of pooling and combinatorial genotyping for detecting very rare variants. As sequencing reads get longer, so will the list of applications.

Tanita Casci

ORIGINAL RESEARCH PAPERS Erlich, Y. et al. DNA Sudoku - harnessing high-throughput sequencing for multiplexed specimen analysis. Genome Res. 19, 1243-1253 (2009)| Prabhu, S. \& Pe'er, I. Overlapping pools for high-throughput targeted resequencing. Genome Res. 19, 1254-1261 (2009) 